\title{
The role of Barents-Kara sea ice loss in projected polar vortex changes
}

\author{
Marlene Kretschmer $^{1}$, Giuseppe Zappa ${ }^{1,2}$, and Theodore G. Shepherd ${ }^{1}$ \\ ${ }^{1}$ Department of Meteorology, University of Reading, Reading, UK \\ ${ }^{2}$ Istituto di Scienze dell'Atmosfera e del Clima, Consiglio Nazionale delle Ricerche, Bologna, Italy
}

Correspondence: Marlene Kretschmer (m.j.a.kretschmer@reading.ac.uk)

Received: 1 July 2020 - Discussion started: 27 July 2020

Revised: 2 November 2020 - Accepted: 5 November 2020 - Published: 20 November 2020

\begin{abstract}
The Northern Hemisphere stratospheric polar vortex (SPV) plays a key role in mid-latitude weather and climate. However, in what way the SPV will respond to global warming is not clear, with climate models disagreeing on the sign and magnitude of projected SPV strength change. Here we address the potential role of Barents and Kara (BK) sea ice loss in this. We provide evidence for a non-linear response of the SPV to global mean temperature change, which is coincident with the time the BK seas become ice-free. Using a causal network approach, we demonstrate that climate models show some partial support for the previously proposed link between low BK sea ice in autumn and a weakened winter SPV but that this effect is plausibly very small relative to internal variability. Yet, given the expected dramatic decrease in sea ice in the future, even a small causal effect can explain all of the projected ensemble-mean SPV weakening, approximately one-half of the ensemble spread in the middle of the 21st century, and one-third of the spread at the end of the century. Finally, we note that most models have unrealistic amounts of BK sea ice, meaning that their SPV response to ice loss is unrealistic. Bias adjusting for this effect leads to pronounced differences in SPV response of individual models at both ends of the spectrum but has no strong consequences for the overall ensemble mean and spread. Overall, our results indicate the importance of exploring all plausible implications of a changing Arctic for regional climate risk assessments.
\end{abstract}

\section{Introduction}

The stratospheric polar vortex (SPV), a band of fast-blowing westerlies forming during boreal winter, is a central component of the Northern Hemisphere atmospheric circulation (Waugh et al., 2016). Variability in vortex strength is not only linked to stratospheric ozone concentrations, but, due to downward coupling to the troposphere, it also strongly affects mid-latitude weather. In particular, extreme states of the SPV are known to influence the phase and persistence of the North Atlantic Oscillation (NAO) and associated storm tracks and weather regimes (Baldwin and Dunkerton, 2001; Kidston et al., 2015).

Understanding potential changes in the SPV in response to global warming is therefore of huge scientific and societal relevance. If the vortex strengthens, for instance, Mediterranean precipitation is expected to strongly decrease, while days of extreme storminess in northern Europe are expected to increase (Simpson et al., 2018; Zappa and Shepherd, 2017). Conversely, if the vortex weakens, the pace of Mediterranean drying is likely to be more moderate and changes in storminess less pronounced.

However, in what way the SPV will respond to a warming climate in the future is highly uncertain. While the multimodel ensemble mean from Phase 5 of the Coupled Model Intercomparison Project (CMIP5) under the RCP8.5 scenario suggests a moderate weakening at the end of the 21 st century, there is huge inter-model spread and no agreement on the sign of change (Manzini et al., 2014; Wu et al., 2019). This remains an issue in CMIP6 (Ayarzagüena et al., 2020).

This spread is not just attributable to different vertical resolutions and model lids but has been speculated to depend 
on distinct wave parametrizations and differently represented dynamical processes (Karpechko and Manzini, 2017; Sigmond and Scinocca, 2010; Wu et al., 2019). Several potential mechanisms influencing SPV strength have been reported in this context, such as El Niño-Southern Oscillation (ENSO) or high-latitude blocking (Domeisen et al., 2018; Martius et al., 2009; Nishii et al., 2010; Peings, 2019), but their relative importance and their role in a changing climate are not well understood (Shepherd, 2014; de Vries et al., 2013). Overall, the future polar vortex change thus remains completely undefined.

Recently, Manzini et al. (2018) reported a non-linear response of the SPV to global mean warming in a single-model large ensemble and hypothesized it to be related to Arctic sea ice loss. More precisely, the SPV was proposed to weaken as long as Barents and Kara sea ice concentrations (BK-SIC) decreased but to strengthen again once the BK seas were icefree (Manzini et al., 2018).

Motivated by their results, we here assess the role of BK sea ice loss for future SPV changes in the CMIP5 ensemble. While the question of whether Arctic sea ice loss contributed to the recent episode of weak vortex events (and associated cold-air outbreaks over Eurasia) is an active area of research (Kim et al., 2014; Kretschmer et al., 2016, 2018; McCusker et al., 2016; Seviour, 2017; Sun et al., 2016), the potential of decreasing Arctic sea ice, and of BK-SIC in particular, to weaken the SPV on longer timescales has been shown in various targeted model experiments (Blackport and Kushner, 2017; Hoshi et al., 2017; McKenna et al., 2017; Nakamura et al., 2016; Screen, 2017a, b; Sun et al., 2015; Zhang et al., 2018a, b). In what way future BK sea ice loss will affect the SPV is, however, not clear (McKenna et al., 2017; Sun et al., 2015). In general, conflicting model and observational results have dominated the scientific debate (Cohen et al., 2020; Screen et al., 2018), with some stressing a likely small and statistically insignificant influence of sea ice on SPV strength (Garfinkel et al., 2017; Seviour, 2017) and on mid-latitude climate (Blackport et al., 2019; Blackport and Screen, 2020) compared to natural variability. As the decline of Arctic sea ice in a warming climate is certain (IPCC, 2014; Notz and Stroeve, 2018), understanding potential impacts on future SPV strength is crucial and forms the aim of the present analysis.

\section{Data}

We use monthly outputs from 35 CMIP5 models (see captions in Fig. 4) for which data are available for our purposes. For each model, the historical (1900-2005) and RCP8.5 (2005-2099) simulations from the same ensemble member are concatenated to produce a continuous time record in the analysed fields. All available ensemble members are considered separately when analysing time series, and their number per model is reported on the $x$ axis of Fig 4 .
For all other analyses, the mean over the available ensemble members per model is calculated first.

For observations of sea ice concentration, we use the latest version (HadISST.2.2.0.0) of version 2 of the Hadley Centre Sea Ice and Sea Surface Temperature data set HadISST.2 (Titchner and Rayner, 2014). Note that this sea ice product gives a rather conservative estimate of monthly sea ice, in particular having higher mean concentrations compared to HadISST.1. For all other variables, we use monthly means of ERA5 reanalysis data as a measure for observations (Hersbach et al., 2020). Analysis for the observations are performed over the period 1979-2018.

Time series are constructed by area-averaging over the respective regions, whereby we include Barents and Kara sea ice concentrations (BK-SIC) over the region $\left(65-85^{\circ} \mathrm{N}\right.$ and $\left.10-100^{\circ} \mathrm{E}\right)($ Screen, 2017a) and sea level pressure over the Ural Mountains region (Ural-SLP; $45-70^{\circ} \mathrm{N}$ and $40-85^{\circ} \mathrm{E}$ ) (Kretschmer et al., 2016) and over the North Pacific (NPSLP; $30-65^{\circ} \mathrm{N}$ and $160-220^{\circ} \mathrm{E}$ ) (Trenberth and Hurrell, 1994). As a proxy for vertical wave activity flux, we compute poleward eddy heat flux $(v T)$ at $100 \mathrm{hPa}$ averaged over 50 $80^{\circ} \mathrm{N}$ (Hoshi et al., 2017). More precisely, the zonal-mean deviations of the monthly mean meridional winds and temperature at $100 \mathrm{hPa}$ at each grid point are first multiplied, and then the spatial mean is calculated. To describe the stratospheric polar vortex (SPV), we follow Wu et al. (2019) and compute the average zonal wind velocity over $60-75^{\circ} \mathrm{N}$ but at $20 \mathrm{hPa}$ instead of $10 \mathrm{hPa}$.

\section{Methods}

\subsection{Changes as a function of global mean warming and BK-SIC loss}

Anomalies of seasonal mean SPV in January, February, and March (JFM), BK-SIC in October, November, and December (OND), and all-year global mean temperature $(T)$ are calculated by subtracting the mean over the reference period 1960-1989. Similar to Manzini et al. (2018), we then calculate a 15-year moving average of global mean temperature change $(\Delta T)$ and include the last 14 years from the historical simulations to calculate the average over the first window. The last year of each window represents this average; that is, the year 2006 represents the mean over 1992-2006. A global warming level is said to be reached when, for the first time, this 15-year average is equal to or larger than a certain threshold level. The SPV change for a given warming level is then calculated as the 30-year average before this warming level was reached. For example, if a warming of $5 \mathrm{~K}$ was reached in the year 2099 (i.e. the global mean temperature change averaged over 2085-2099 exceeds 5 K), SPV change is calculated over the period from 2070 to 2099 . We proceed equivalently when plotting BK-SIC and $v T$ change as a 
function of temperature change and when plotting SPV and $v T$ change as a function of BK-SIC change.

\subsection{Estimating the timing of an ice-free BK}

The year the BK seas become ice-free is here defined as the first year that projected BK-SIC anomalies (relative to the reference period 1960-1989) fall below $5 \%$ of the observational mean of 0.51 (calculated over the same reference period). This refers to the fraction of BK-SIC being lower than 0.026 ; i.e. less than $2.6 \%$ of the BK seas are covered with sea ice. For models that are not ice-free before the end of the 21st century, we calculate the expected year they will become ice-free by fitting a linear trend line over the years 2006-2099. The first year this trend line is below 0.026 is then defined as the year the model's BK seas are expected to become ice-free (see Fig. 1d).

\section{Results}

\subsection{The non-linear response of the polar vortex}

To test for evidence of a non-linear SPV response related to sea ice loss in the CMIP5 models, we plot the projected SPV change in January-March (JFM), the projected $v T$ change in December-February (DJF), and BK-SIC change in OctoberDecember (OND) for different levels of global mean warming in the RCP8.5 high-emissions scenario (Fig. 1a-c). We show the evolution of each model (grey lines), as well as the multi-model mean (blue lines), with darker shades of blue indicating means over the subset of models with stronger warming at the end of the 21 st century.

The ensemble-mean SPV weakens by up to approximately $2 \mathrm{~m} \mathrm{~s}^{-1}$ for $2.5 \mathrm{~K}$ warming and strengthens slightly afterwards for models reaching $5 \mathrm{~K}$ warming at the end of the century (see dark blue line in Fig. 1a) or remains constant (lighter blue lines in Fig. 1a). Consistently, the ensemble-mean poleward eddy heat flux $v T$ first increases and then plateaus with global-mean warming exceeding $2.5 \mathrm{~K}$ (Fig. 1b). This coincides with a flattening of the multi-model mean BK-SIC change (Fig. 1c), indicating that the BK seas have become ice-free in several models (see dark blue line and upper bounds of the ensemble spread in Fig. 1c). In contrast, when plotting SPV change and $v T$ change as a function of BK-SIC change, we find them to be approximately linear, with most models showing a weakening of the SPV and a strengthening of $v T$, while BK-SIC decreases (Fig. 1d and e). These results (Fig. 1a-e) are robustly found when plotting the multi-model median instead of the mean (not shown), indicating that they are not just the result of a few outliers.

Interestingly, not only does the maximum temperature change vary across models as a result of different climate sensitivities, but also the amount of BK-SIC loss varies substantially because of different BK-SIC climatologies. In fact, the timing of an ice-free BK (see methods) can be well predicted from the model's BK-SIC climatology divided by the projected global mean warming at the end of the 21st century ( $r=0.82$; Fig. 1f). For $66 \%$ of the models, the BK seas are ice-free in OND before the year 2100. This includes, in particular, all but one model with below-average sea ice conditions compared to observations. Models with excessive initial sea ice, in contrast, are, on average, ice-free later.

To test for a role of the timing in BK becoming ice-free in the non-linearity of the SPV response, we next calculate the multi-model mean response of SPV, $v T$, and BK-SIC to global mean temperature change separately for models which are ice-free early (before the year 2090; thick blue lines in Fig. 2a-c) and late (after the year 2090; thick grey lines in Fig. 2a-c). The non-linearity we found for the full ensemble is only present in the former group of models. In contrast, the models which keep losing BK sea ice over almost the entire period (grey lines in Fig. 2a) also show ongoing weakening of the SPV (grey lines in Fig. 2a) and strengthening of $v T$ (grey lines in Fig. 2b) with global mean warming.

To further account for the spread regarding the timing of BK-SIC being gone, we show the 30-year running mean SPV change, which is aligned and normalized with reference to the year the BK seas become ice-free (Fig. 2d). Thus, by construction, all time series have value 1 at year 0 (the year BK-SIC is gone). For consistency with Figs. 1a-e and $2 \mathrm{a}-\mathrm{c}$, the SPV change is evaluated relative to the 1960-1989 period, but our results are not sensitive to the chosen start period. To aid visualization, start and end values are highlighted with dots. Before the BK seas are ice-free (grey lines and dots), values above 1 thus indicate a weakening with time of the SPV, while they imply strengthening afterwards (blue lines and dots). Up to the time the BK seas are ice-free, the SPV weakens in two-thirds of the models. Afterwards, only a few models show further weakening, while most indicate a strengthening SPV (values above 1) or no further change (values close to 1). Thus, there is an indication of a weakening signal of the SPV in CMIP5 models up to the point where BK-SIC is gone, with the response switching sign thereafter.

This difference in SPV change before and after the BK seas are ice-free is further shown in a box and whisker plot (Fig. 2e). As we compare changes over different time intervals, we divide the SPV change by the model's global mean temperature change over the considered time span. The CMIP5 ensemble shows a robust weakening SPV signal before BK-SIC is gone with most of the inter-quartile range being below zero (grey box plot). For those models for which sea ice is gone before the end of the 21st century, the SPV strengthens on average. In particular, 14 out of the 18 early ice-free models show a positive difference in SPV change before and after the time the BK seas have become ice-free. According to a binomial distribution, the probability of this occurring by chance is only 0.01 , thus indicating a role of BK-SIC loss. 
(a)

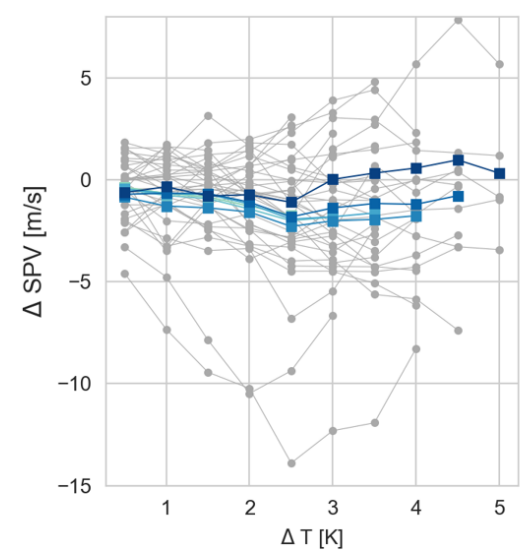

(d)

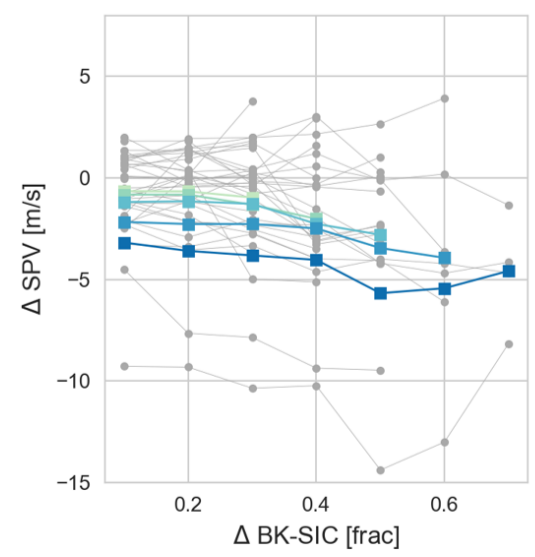

(b)

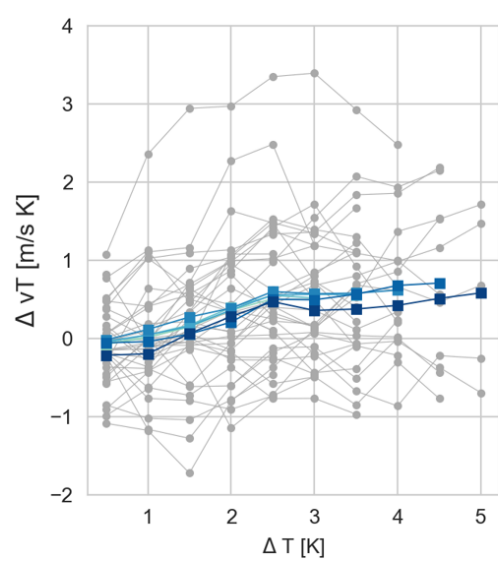

(e)

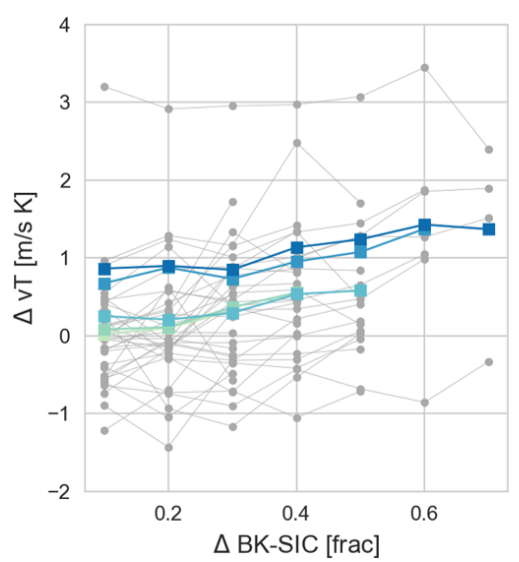

(c)

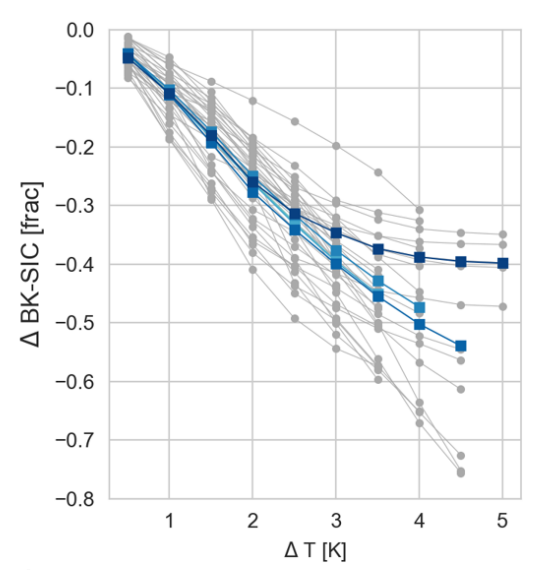

(f)

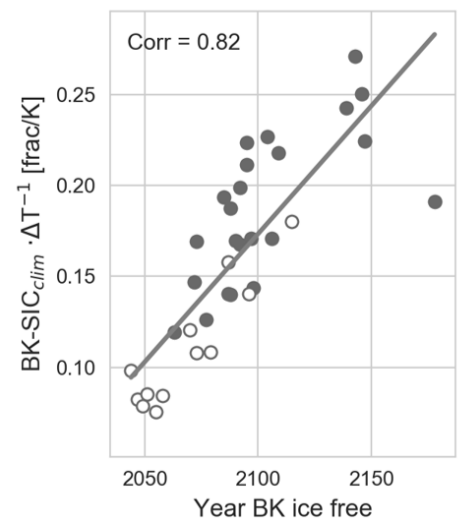

Figure 1. Non-linear response of the SPV to global warming. (a) SPV change in winter (January, February, March; JFM) as a function of annual mean global mean temperature change in the CMIP5 models (see methods). The thick blue lines show the ensemble mean for different sub-sets of the warming level reached at the end of the 21 st century, with darker colours indicating higher warming levels. For example, the darkest blue line shows the ensemble mean for the set of models reaching $5 \mathrm{~K}$ warming, while the second darkest line is the mean over all models reaching at least $4.5 \mathrm{~K}$ warming (thus including those models also reaching $5 \mathrm{~K}$ warming). (b) Same as (a) but for $v T$ change (December, January, February; DJF). (c) Same as (a) but for BK-SIC change in autumn (October, November, December; OND). (d) Same as (a) but for SPV change as a function of BK-SIC change. (e) Same as (b) but for $v T$ change as a function of BK-SIC change. (f) Estimated timing of an ice-free BK in OND versus its climatology in 1960-1989 divided by global mean temperature change at the end of the 21 st century, i.e. the warming averaged over the 2070-2099 period. The dark circles indicate the models that have too much BK-SIC compared to observations and the open circles those with too little sea ice.

Overall, consistent with the single-model large ensemble results of Manzini et al. (2018), Figs. 1 and 2 thus suggest a non-linear response of the SPV in CMIP5 models to global mean warming which is dependent on the timing of when the BK seas become ice-free. The non-linearity in the lower stratospheric $v T$ response further suggests that this SPV nonlinearity originates in the troposphere.

\subsection{Potential confounding factors}

In the following, we aim to understand the contribution of BK-SIC change to SPV change in more detail. This is challenging given a fully coupled climate system with several likely competing effects which might both reinforce or di- lute the signal of interest. In this context, naive regression or correlation analyses have been questioned with respect to their causal interpretation as they exhibit several limitations (Blackport et al., 2019; Kretschmer et al., 2016).

For example, common drivers can spuriously increase the regression strength or may even, if the influence is of the opposite sign, dilute the true relationship between two processes (Kretschmer et al., 2016). Further, the autocorrelation of a time series, which is characteristic of sub-seasonal Arctic sea ice concentrations or polar vortex strength, inflates the correlation strength, potentially suggesting a false link between two processes (Runge et al., 2014; McGraw and 
(a)

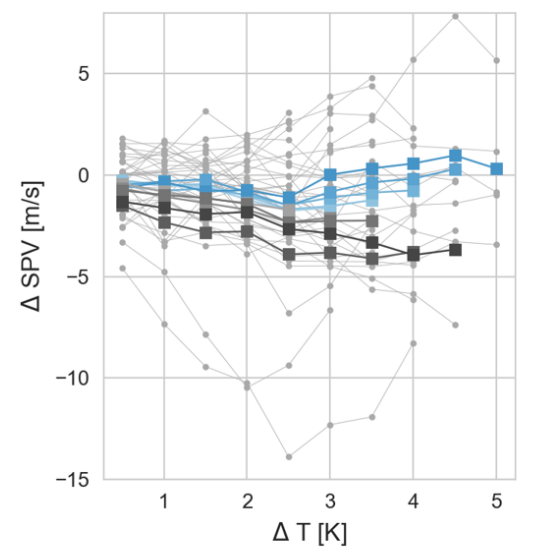

(d)

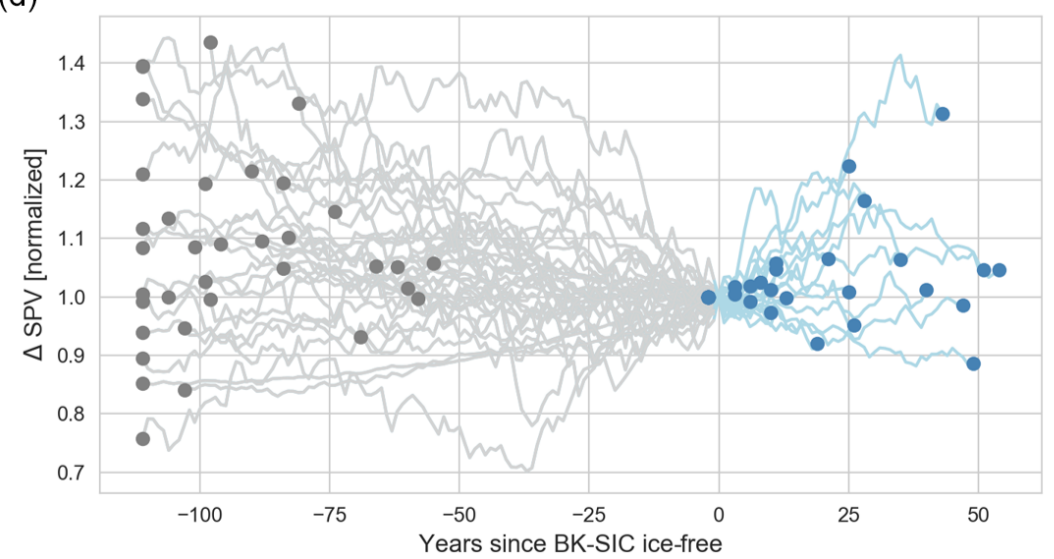

(b)

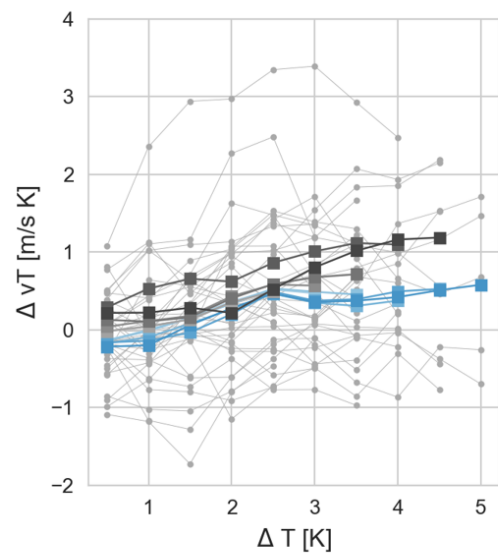

(c)

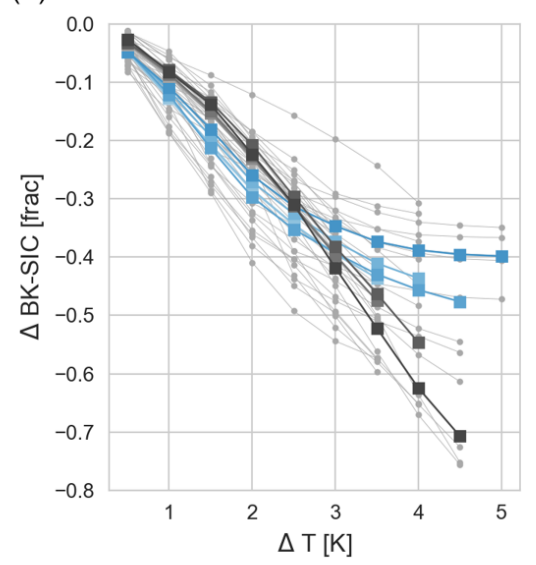

(e)

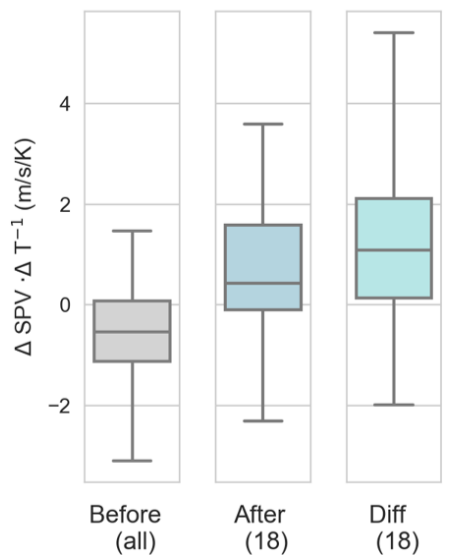

Figure 2. The role of the timing of the BK seas becoming ice-free. (a-c) As in Fig. 1a-c but with the ensemble mean indicated separately for the subsets of models where the BK seas become ice-free early (before 2090, blue lines) and those which are ice-free late (after 2090, dark grey lines). (d) Time series of moving 30-year mean SPV normalized by the 30-year mean reached before the BK seas become ice-free (in OND). Grey lines thus show the evolution of change when there is sea ice and blue lines for when BK is ice-free. Dots indicate the values at the end (blue) and at the start (grey). (e) Boxplot of SPV change before and after BK is ice-free (normalized by global mean warming level over the respective period), as well as the difference for each model. The boxes indicate the inter-quartile range, and the whiskers represent the upper and lower quartile ranges. Only models which were ice-free for at least 10 years (i.e. before 2090) are included in the latter two boxplots (in total 18 models). In all panels, changes were calculated relative to the 1960-1989 reference period (see also Sect. 3).

Barnes, 2018). Thus, to estimate the influence of BK-SIC on SPV, one has to control for such factors.

To do this, we need to assume a causal model of the underlying processes, here shown in the form of a graphical network (Fig. 3). Nodes represent different sub-seasonal processes, and the arrows indicate causal relationships between them with arrows self-connecting to nodes representing the auto-dependence of that process. This network can be interpreted as our attempt to summarize the large body of literature on the topic in the most parsimonious way, recognizing it as being prone to subjective judgement and only representing a reduced model of the underlying truth.

A reduction in Barents and Kara sea ice concentrations in autumn and early winter is assumed to increase turbulent heat flux in this region, leading to enhanced sea level pressure over the Ural Mountain region (Ural-SLP), as shown by sev- eral studies (Kim et al., 2014; Kug et al., 2015). Via constructive interference with the climatological stationary wave, this enhances the vertical wave activity flux $(v T)$ into the stratosphere, causing a weakening of the vortex (SPV) in winter (Kretschmer et al., 2016; Peings, 2019). However, Ural-SLP also affects BK-SIC (Blackport et al., 2019; Tyrlis et al., 2019), making it hard to isolate the signal emerging from sea ice alone. Further, tropical Pacific variability, e.g. in the form of the El Niño-Southern Oscillation or the Madden-Julian Oscillation (ENSO/MJO), can affect $v T$ and thus the SPV via altered sea level pressure anomalies over the North $\mathrm{Pa}$ cific (NP-SLP) (Domeisen et al., 2018). Furthermore, sea ice decline in the North Pacific (NP-SIC) has also been causally linked to lower NP-SLP and thus a strengthened SPV (Kug et al., 2015). As NP-SLP can also affect Ural-SLP via Rossby wave propagation, it confounds the analysis of the BK-SIC to 


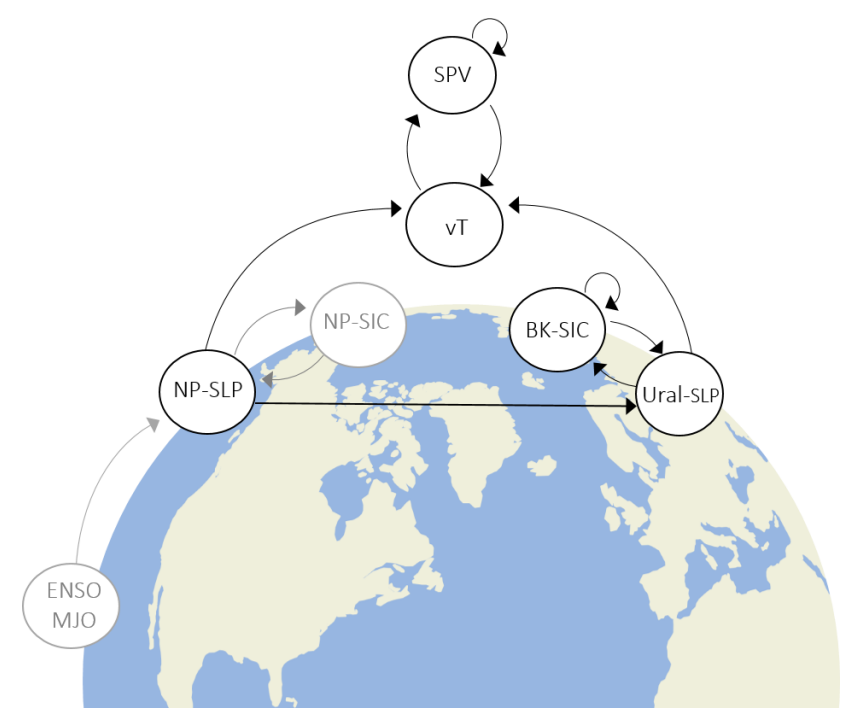

Figure 3. Assumed causal model. Nodes in black contours represent the involved processes in the causal model: Barents and Kara sea ice concentrations (BK-SIC), sea level pressure over the Ural Mountains (Ural-SLP) and over the North Pacific (NP-SLP), lower-stratospheric poleward eddy heat flux $(v T)$, which is the upward wave forcing of the stratospheric circulation, and the stratospheric polar vortex (SPV). The black arrows represent the corresponding causal relationships which are here assumed to operate on a monthly timescale. The grey contoured nodes of the North Pacific sea ice concentrations (NP-SIC) and El Niño-Southern Oscillation/Madden-Julian Oscillation (ENSO/MJO) and the respective arrows denote processes discussed in the literature but not explicitly accounted for here because their effects are assumed to be mediated by NP-SLP.

SPV pathway (Jiménez-Esteve and Domeisen, 2018; Warner et al., 2020).

Note that downward stratosphere-troposphere coupling is not included here as we consider tropospheric processes in autumn and early winter only with the downward links, e.g. from SPV to Ural-SLP or BK-SIC, expected in middle and late winter (Kidston et al., 2015; Smith et al., 2018). That is because our interest here is in understanding the response of SPV to global warming.

\subsection{Estimating the causal effect of BK-SIC on SPV}

Making our assumptions of the underlying causal structure explicit has the advantage that it guides further statistical analyses. We next try to quantify the (indirect) influence of autumn BK-SIC on winter SPV in the historical simulations. We recognize that an exact quantification is probably not possible due to the large internal variability, including a documented intermittency of the BK-stratosphere pathway (Siew et al., 2020) and Arctic-midlatitude linkages (Kolstad and Screen, 2019; Overland et al., 2016), as well as uncertainties regarding the involved time lag (Blackport and Screen, 2019;
García-Serrano et al., 2017). Our aim, therefore, is to come up with a plausible estimate of the mean causal effect.

To achieve this, according to causal inference theory, it is sufficient to "block" all confounding processes (Pearl, 2013), i.e. processes that influence both autumn BK-SIC and winter SPV. Assuming linear dependence, this can be done by regressing the seasonal-mean winter (JFM) SPV on late autumn (OND) BK-SIC and, to control for confounding, additionally regress on autumn (SON) Ural-SLP:

$\mathrm{SPV}_{\mathrm{JFM}}=a \mathrm{BK}-\mathrm{SIC}_{\mathrm{OND}}+b \mathrm{Ural}_{-\mathrm{SLP}} \mathrm{SON}$,

whereby $a$ is interpreted as the mean causal effect of autumn BK-SIC on winter SPV. For consistency with Fig. 1, we used seasonal-mean data and therefore do not control for autocorrelation. We explicitly do not control for winter (DJF) Ural-SLP and $v T$ as the influence of BK-SIC is assumed to be mediated by these variables (Fig. 3), and we would thus regress out exactly the pathway we aim to measure. Note further that the confounding effect of NP-SLP is mediated by Ural-SLP and thus accounted for. Yet, even when including autumn NP-SLP in the regression, our results are only marginally affected.

To account for sampling uncertainties and facilitate comparison with observations, we calculate the regression over different 39-year-long moving windows over the historical simulations from 1900 to 2005, resulting in 67 partly overlapping windows overall. As we compare models with different SPV and BK-SIC variabilities, the regressions were performed over standardized time series by first subtracting the mean and then dividing by the standard deviation for each season. Both mean and standard deviation are calculated over the considered 39-year time window. Further, linear trends are removed by fitting a regression slope over the time series of each window.

Figure 4a shows the spread of the regression parameter $a$ for different models and time windows (left panel), indicating large intra-model and inter-model spread. Yet, as expected, most models have a positive mean causal effect (right panel in Fig. 4a) with a median of 0.035. The histogram of all link strengths (middle panel in Fig. 4a) shows a bell-shaped distribution with a positive mean of 0.052 , meaning that, on average, a change by 1 standard deviation $(\sigma)$ in BK-SIC leads to a $0.052 \sigma$ change in SPV.

When regressing out the effect of OND (instead of SON) Ural-SLP, the ensemble-mean causal effect reduces slightly to 0.036 . In contrast, we get slightly higher regression coefficients when averaging BK-SIC over November and December and SPV over January and February only (not shown). Results are also similar when using monthly time series and additionally controlling for the autocorrelation of BK-SIC. Overall, albeit a weak signal, there is thus evidence for less BK-SIC in autumn causing a weakening of the SPV in winter under the premise of the causal model being true.

Note that the causal effect for the observations is as high as 0.38 , which is on the outer tail of all computed link 
(a)

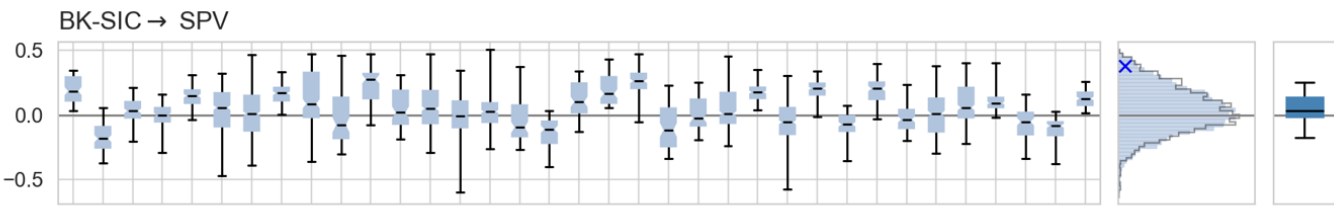

(b)

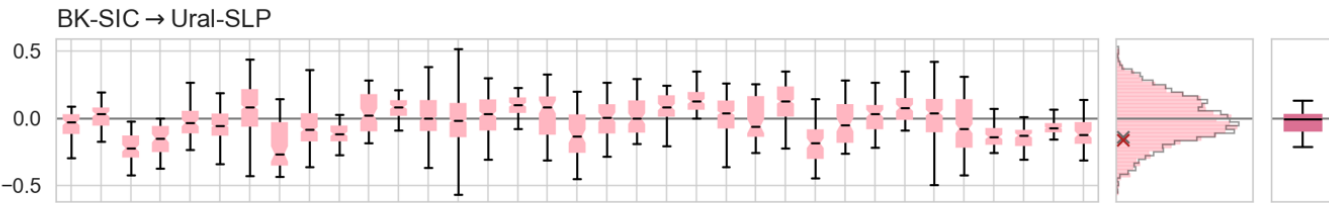

(c)

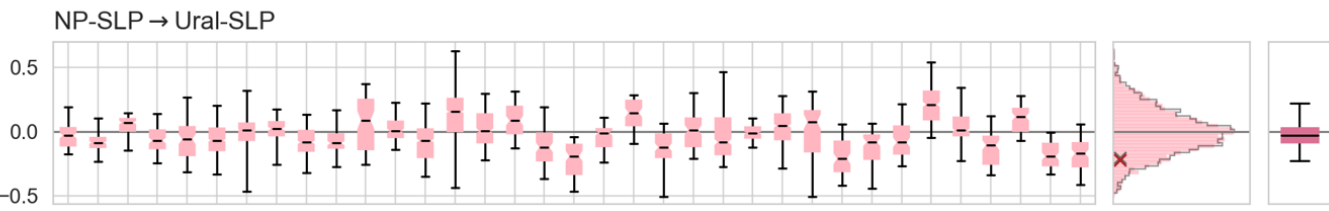

(d)
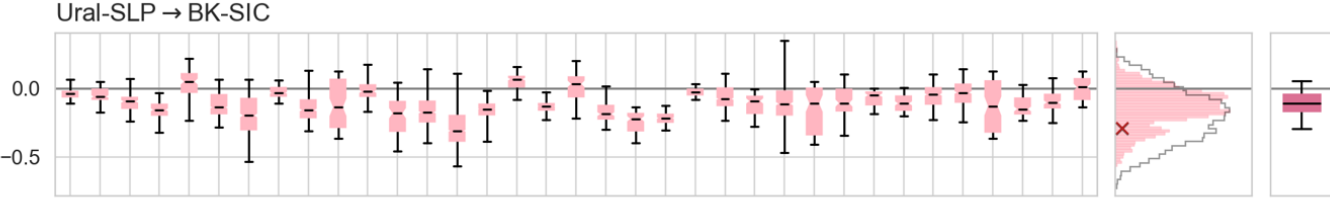

(e)

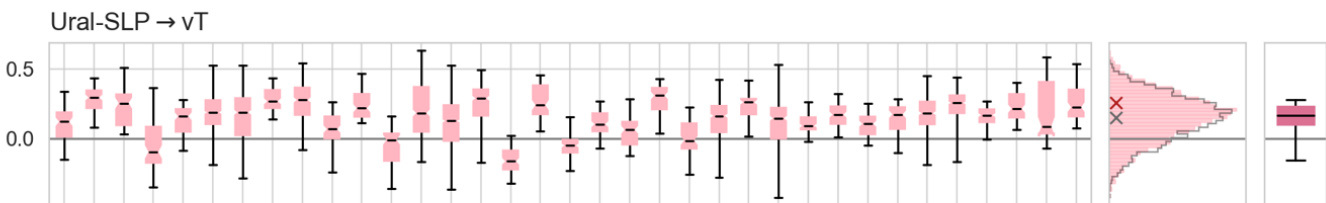

(f)

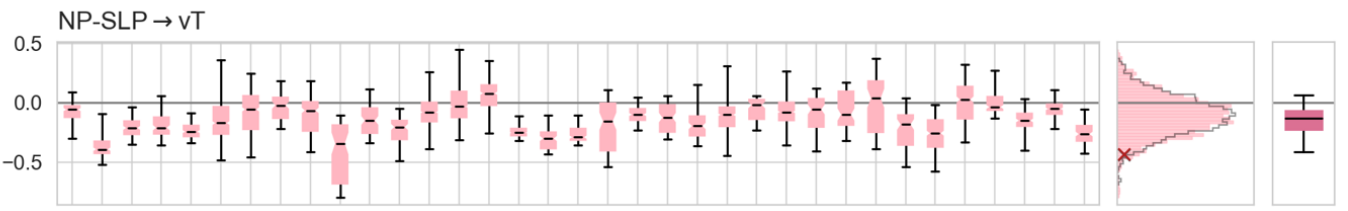

(g)

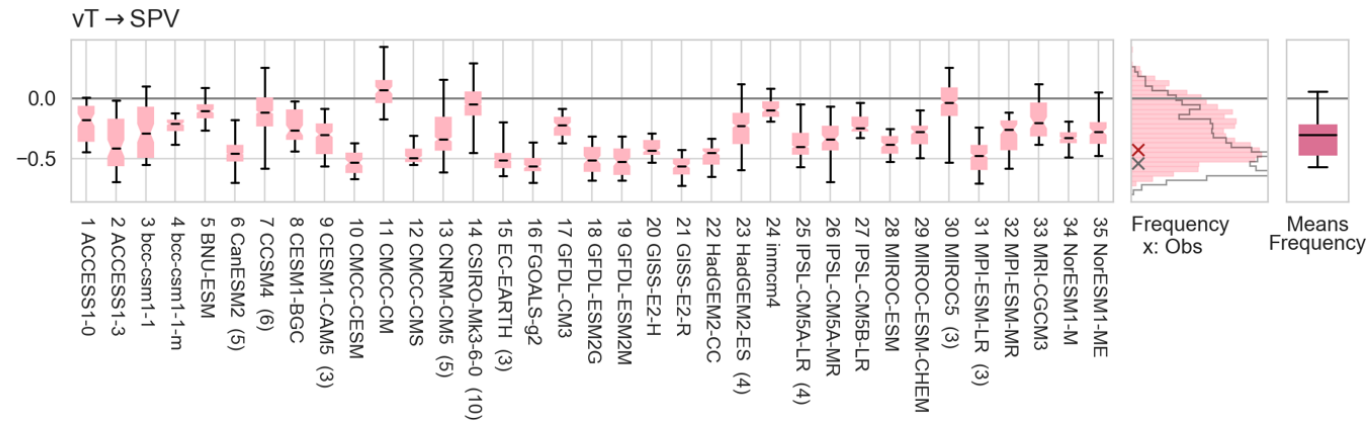

Figure 4. Individual links of Arctic-stratosphere pathway. Links from (a) seasonal-mean BK-SIC (in OND) to SPV (in JFM) and from (b) monthly BK-SIC (in N) to Ural-SLP (in J), (c) NP-SLP (in D) to Ural-SLP (in J), (d) Ural-SLP (in N) to BK-SIC (in D), (e) Ural-SLP (in D) to $v T$ (in J), (f) NP-SLP (in D) to $v T$ (in J), and (g) $v T$ (in J) to SPV (in F). What is shown is the spread in link strength for each model (left panels) and the distribution of all link strengths (middle panels) and of the models' means (right panels). The link strengths are quantified by regressing each variable on their parents (see Fig. 3) for each model over 39-year moving windows from 1900 to 2005 (in total 67 windows) in the historical simulations. Grey contours in the middle panel show the histogram obtained using (unadjusted) regression. The crosses in the middle panel denote the link strength obtained using observations (grey for unadjusted regression and coloured crosses for regressions including all parents). Numbers in brackets after model names on the $x$ axis indicate the number of ensemble members used, with no number meaning that just one member was used. The box and whisker plots thus include different amounts of data (number of ensemble members times the 67 moving windows). The boxes indicate the inter-quartile range, the whiskers represent the upper and lower quartile ranges, and horizontal lines show the median. 
strengths (blue cross in middle panel in Fig. 4a). Previous studies suggested that models systematically underestimate the effect emerging from BK-SIC (Cohen et al., 2020). On the other hand, this potential discrepancy between models and observations was also attributed to a relatively active stratospheric pathway over recent years (Siew et al., 2020) and therefore is not representative of the actual, likely much lower link strength. Our results could be interpreted in both ways, but addressing this aspect lies outside the scope of the present analysis.

\subsection{Representation of the BK-SIC to SPV pathway}

In an attempt to better understand the inter-model spread, we further compute the link strengths of the assumed mediating processes. This can be done by regressing each process $\mathrm{Y}$ on its ingoing links (Fig. 3) with the regression coefficients interpreted as the respective link strength (Pearl, 2013).

For example, to estimate the effect of November BK-SIC on January Ural-SLP, we compute the following:

Ural-SLP $_{\mathrm{J}}=a \mathrm{BK}-\mathrm{SIC}_{\mathrm{N}}+b \mathrm{NP}-\mathrm{SLP}_{\mathrm{D}}$,

with $a$ denoting the causal effect of BK-SIC (Fig. 4b) on Ural-SLP and $b$ that of NP-SLP (Fig. 4c). Both effects are found to be very weak on these monthly timescales and can only be seen in roughly half of the model averages with no signal in the multi-model median (right panels in Fig. 4b and c). In a similar way, we also compute the influence of November Ural-SLP on December BK-SIC (Fig. 4d), of December Ural-SLP and NP-SLP on January $v T$ (Fig. 4e and f), and of January $v T$ on February SPV (Fig. 4g). Though the spread in link strength within and across models is again large, they are mostly of the expected sign, and results are robust when choosing different winter months.

The weak or missing mediated signal from BK-SIC to Ural-SLP illustrates a dilemma that is frequently faced when studying the impact of sea ice on mid-latitude circulation. On the one hand, if our null hypothesis was the non-existence of such a link, we could not reject it based on the presented results (avoidance of type- 1 error). On the other hand, as failure to reject a hypothesis does not prove the hypothesis, we cannot rule out the possibility of an influence of BK-SIC on SPV via Ural-SLP (avoidance of type-2 error). For example, our choices on the regional indices and the timescales and time lags might not be optimal, and/or be model-dependent, hence diluting the mean signal.

Some models seem to be systematically underestimating the assumed BK-SIC to SPV pathway. For example, model 16 (FGOALS-g2) is a notable outlier for the link from Ural-SLP to $v T$ (Fig. 4e). However, as the sample sizes are small and the analyses only represent proxies and "snapshots" of the studied links, there is no obvious justification for excluding models on this basis to reduce the spread in future SPV projections. Doing this would require a more de- tailed analysis of the processes and timescales in the individual models.

Overall, we can thus neither prove nor disprove the representation of the individual chain of mediating links in the historical simulations of the CMIP5 ensemble. However, rejecting our initial assumption of a causal link from BK-SIC to SPV would leave us with the problem of explaining the non-linear SPV response to global warming, as presented in Figs. 1 and 2. Therefore, our approach will now consist of exploring the implications for the SPV under climate change, assuming that a weak signal from BK-SIC to SPV indeed exists as suggested by previous studies (De and Wu, 2019; Kim et al., 2014; Screen, 2017b; Zhang et al., 2018b) and supported by Fig. 4a. In other words, our approach is primarily one of deduction rather than of induction.

\subsection{Implications for projections of SPV change}

Addressing possible implications of future BK sea ice loss for SPV change seems particularly justified given an expected decrease of sea ice under global warming (Notz and Stroeve, 2018), making it necessary to assess related risks (Sutton, 2019). Further, the results presented in Figs. 1 and 2 indicate a role of BK-SIC in SPV change, which we try to understand.

To do this, we test how well the projected BK-SIC changes (relative to 1960-1989) can explain the projected SPV changes across the RCP8.5 simulations for different assumed standardized causal effects (ces) of 0.025, 0.05, and 0.1 . These levels are motivated by the regression strength found over the historical period (Fig. 4a) representing plausible estimates of the mean causal effect of BK-SIC on SPV. For example, 0.05 is about the ensemble-mean regression strength (0.052), 0.025 is slightly below the ensemble median (0.035), and 0.1 is slightly below the upper quartile range (0.14). Furthermore, using only one (standardized) effect for the whole ensemble seems reasonable as there is no strong evidence of models having very different behaviours (Fig. 4).

To express ce in physical units and to account for different variability in the different models, we weigh it by ratios of standard deviations ( $\sigma$, calculated over the reference period 1960-1989) for each model $m$ :

$\mathrm{CE}_{\mathrm{m}}=\mathrm{ce} \cdot \sigma_{\mathrm{SPV}_{\mathrm{m}}} \cdot \sigma_{\mathrm{BK}-\mathrm{SIC}_{\mathrm{m}}}^{-1}$.

The distribution of these values for ce $=0.05$ over the different models is shown in Fig. 5a. Note that seasonalmean (OND) and individual monthly variability are comparable for most models. Only for model 5 (BNU-ESM) and model 27 (IPSL-CM5A-MR) does this not hold as these models have basically constant sea ice conditions in December. In the following, these models are therefore excluded from the presented results, but including them does not change our main results. 
(a)

Causal Effect BK-SIC $\rightarrow$ SPV

$\mathrm{ce}=0.05, \mathrm{CE}_{m}=\mathrm{ce} \cdot \sigma_{\mathrm{SPV}} \cdot \sigma_{B K-S I C_{m}}^{-1}$

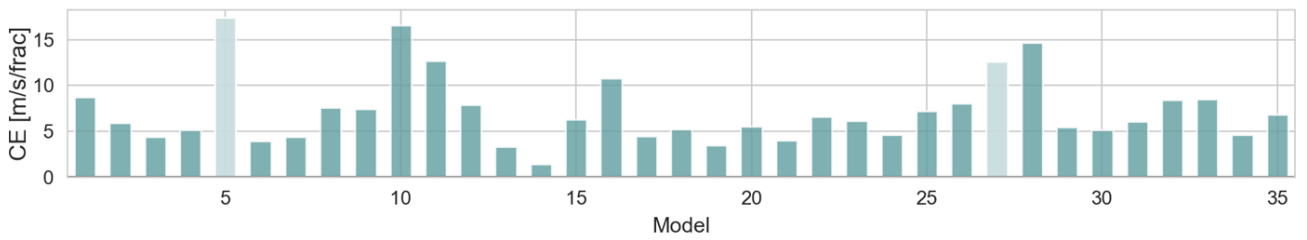

(b)

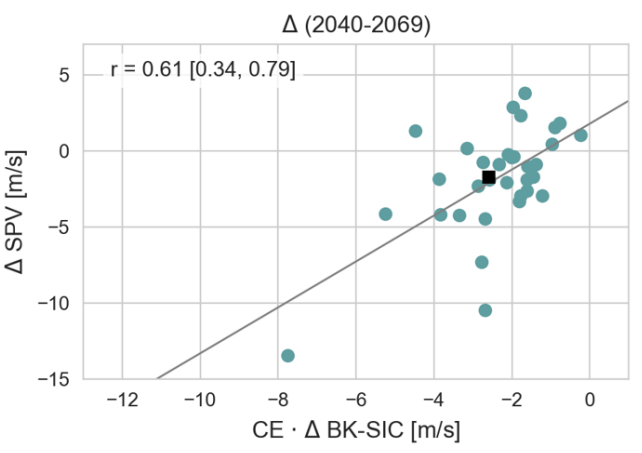

(d)

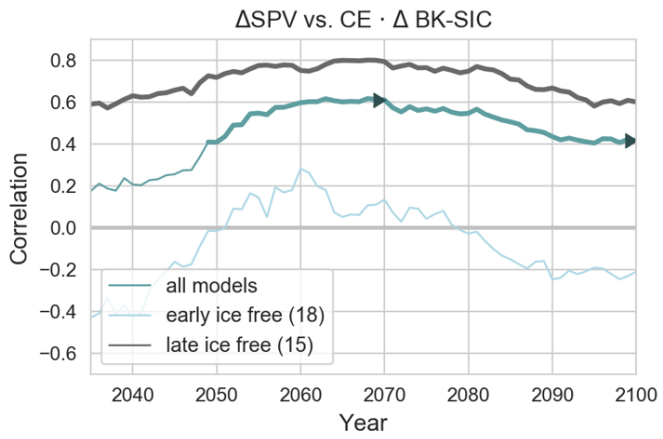

(c)

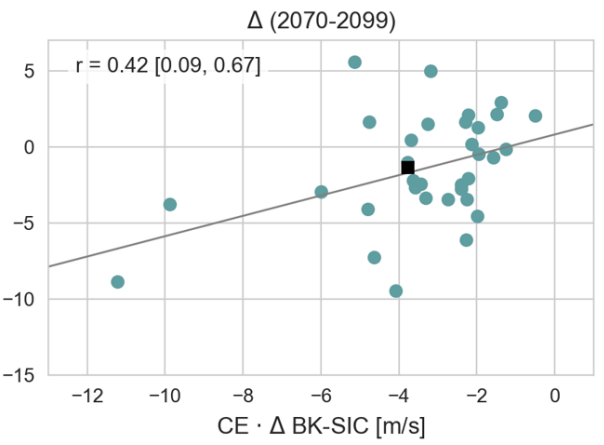

(e)

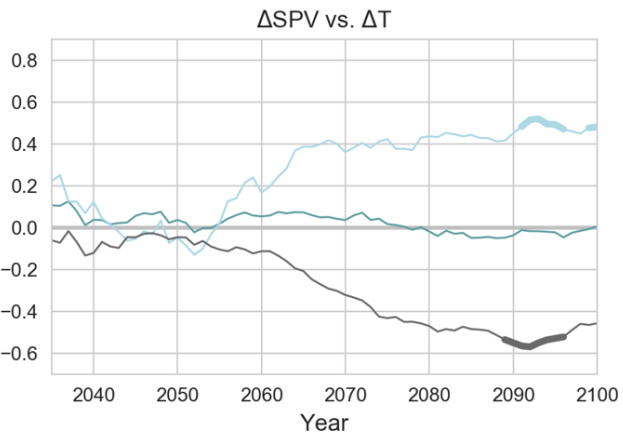

Figure 5. Predicted versus projected polar vortex changes. (a) Causal effect weighted by ratios of standard deviations of BK-SIC and SPV (calculated over the 1960-1989 reference period) to transform standardized ce $=0.05$ into physical units. (b) Projected versus predicted winter (JFM) SPV changes for mid-century (2040-2069). Prediction based on autumn (OND) sea ice. Each dot indicates one model. Changes are calculated relative to 1960-1989. Squares show ensemble means, $r$ denotes the correlation coefficient, and numbers in brackets show the $95 \%$ confidence interval. (c) Same as (b) but for end-of-century (2070-2099) change. (d) The green line shows the correlation (predicted versus projected SPV changes) but for different moving windows. The years on the horizontal axis denote the last year of the 30-year average. The grey line shows the same but only for models for which BK-SIC is not ice-free before 2090 and the blue line for models that are ice-free before 2090. Thick parts of the lines indicate statistically significant correlation values $(p<0.05)$ according to a two-sided Student's $t$ test. (e) Same as (d) but for SPV change versus global mean temperature change.

We next show the scatter plots of predicted winter (JFM) SPV change based on autumn (OND) BK-SIC change versus the actual projected SPV change for mid-century (20402069; Fig. 5b) and end of century (2070-2099; Fig. 5c). For the former period, the statistically predicted and actual projected SPV changes correlate significantly ( $r=0.61$, $p<0.01$ according to a two-sided Student's $t$ test). This correlation is independent of the chosen causal effect strength but is a result of the correlation between BK-SIC and SPV change $(r=0.4)$, which increases after accounting for the different ratios of standard deviations between models.
Assuming a causal effect of 0.05 , the prediction model explains $42 \%$ of the ensemble spread (measured in median absolute deviation, MAD), and the ensemble mean predicts a SPV change of $-2.4 \mathrm{~m} \mathrm{~s}^{-1}$ compared to an ensemble-mean projected SPV change of $-1.8 \mathrm{~m} \mathrm{~s}^{-1}$. Thus, the BK-SIC decrease can account for all of the projected mid-century SPV weakening (and beyond) and almost half of the ensemble spread. For the end-of-century prediction (Fig. 5c), the correlation is still statistically significant $(p<0.01)$ but drops to 0.42 , while the model still explains $38 \%$ of the ensemble spread and overestimates the mean change by about a factor 
of 2. For assumed causal effects of 0.025 and 0.1 , the explained mean and variance halve and double, respectively.

Building upon our initial analysis (Figs. 1 and 2), we hypothesize that the drop in correlation and overestimation of the mean SPV change between the middle and end of the century is due to more models having ice-free BK seas at the end of the century. This is tested by calculating the correlation of predicted and projected SPV changes over different moving windows within the 21 st century (Fig. 5d). For the 15 models that are ice-free late (after the year 2090), the predicted and projected SPV changes correlate strongly (up to 0.8 ) over the entire century. In contrast, models that are ice-free early (before 2090) only show a moderate correlation which is dropping and becoming negative in the second half of the century. This makes sense as models that are ice-free late have more and longer lasting BK-SIC (Fig. 2c), and thus the effect on SPV is more dominant. For models that are ice-free early, in contrast, the BK-SIC effect on SPV change disappears once the BK-SIC forcing is gone.

Consistent with Fig. 1a, we find no correlation between SPV and global mean temperature change (turquoise line in Fig. 5e). However, this may stem from the fact that models that are ice-free in the BK seas early and late have correlations of opposite signs. The moderate positive correlation (up to 0.5) of global mean temperature and SPV change for early ice-free models supports the initial hypothesis that once BK-SIC is gone, it ceases to exert an effect, and the SPV strengthens in response to global mean warming.

\subsection{The tug of war over future SPV change}

The estimated causal effect from BK-SIC to SPV allows us to decompose the projected SPV response to global warming (Fig. 6a and d) into a contribution from BK-SIC change (Fig. $6 \mathrm{~b}$ and e) and all remaining factors (Fig. $6 \mathrm{c}$ and f). The latter is simply calculated as the residuals, i.e. projected minus predicted SPV, and can be interpreted as the effect of global mean warming on the SPV without the effect mediated via BK-SIC loss. We show the evolution of the three quantities as a function of both time (Fig. 6a-c) and global mean temperature change (Fig. 6d-f).

First we test whether such a decomposition provides any evidence against our assumption of BK-SIC being a source of non-linearity of the SPV response to global mean warming. To quantify this, we calculate the Bayes factor for each model; that is, we calculate the odds of observing the data under the alternative hypothesis of future changes in SPV not depending on BK-SIC against that of observing the data under our initial hypothesis of BK-SIC playing a role (with ce in the range of 0.025 and 0.1). Details of the calculations are provided in the Supplement. A Bayes factor much larger than 1 would mean that the data were much more likely under the alternative hypothesis and would force us to question our assumption. However, we find the Bayes factor to be close to unity for almost all models (see also Fig. S1 in the Sup- plement), meaning that their results are equally likely under the two hypotheses. While this analysis does not prove our hypothesis of an influence of BK-SIC on the non-linearity of the SPV response (which rests on Figs. 1 and 2, together with previous literature), neither does it provide any evidence against the assumption. Thus, it is reasonable to retain our hypothesis, justifying our deductive approach.

When assuming $c e=0.05$, the effect of BK-SIC loss on SPV change at the end of the century ranges from almost no change up to a weakening of more than $10 \mathrm{~m} \mathrm{~s}^{-1}$, with the ensemble mean predicting a change of $-3.4 \mathrm{~m} \mathrm{~s}^{-1}$ (Fig. 6b). Thus, even for a small standardized causal effect of 0.05 , the projected dramatic decrease in BK sea ice implies relatively large SPV changes (Fig. 6b and e). In fact, all of the projected ensemble-mean SPV changes (thick lines in Fig. 6a and d) can be explained by a BK-SIC decrease with the residual's ensemble mean being close to zero for the first half of the century and for global mean warming up to $2.5 \mathrm{~K}$ (Fig. $6 \mathrm{c}$ and f). After the year 2060, the residual's ensemble mean even becomes positive, and most ensemble members indicate a strengthened SPV for a global mean temperature change above $2.5 \mathrm{~K}$ (Fig. $6 \mathrm{c}$ and $\mathrm{f}$ ). Thus, if BK-SIC did not decrease, a moderate strengthening of the SPV in response to global mean warming would be expected (Fig. 6f), which appears to be approximately linear.

For a doubled causal effect (ce $=0.1)$ of BK-SIC on SPV, the residual's ensemble mean would imply a strengthening of more than $5 \mathrm{~m} \mathrm{~s}^{-1}$ at the end of the century (dashed line in Fig. 6c). For a causal effect of only 0.025, in contrast, it basically implies no SPV change over the 21 st century in the residuals (dotted line in Fig. 6c).

In summary, Fig. 6 shows the opposing effects, often called a "tug of war", on the SPV of climate change manifested by BK-SIC decrease and other effects not specified here. Note that these other effects could also include potential effects of Arctic sea ice loss in regions other than the BK seas (McKenna et al., 2017; Sun et al., 2015). While a BK-SIC decrease accounts for all the projected ensemblemean SPV weakening even for a small assumed ce of 0.025 , the effect not mediated by BK-SIC ranges from no change (for ce $=0.025$ ) to a pronounced strengthening (for ce $=0.1$ ).

\subsection{Bias-adjusted sea ice concentrations}

Finally, we note that most CMIP5 models have too much initial BK-SIC compared to observations (Fig. 7a; see also Fig. 1f) meaning that their SPV response to sea ice loss is too strong. The opposite holds for models with too little initial BK-SIC. We therefore include a simple bias-adjustment function to estimate the expected SPV change for more realistic sea ice conditions.

Figure $7 \mathrm{~b}$ indicates for each model the periods before and after the BK seas have become ice-free, as well as the periods when a bias adjustment is needed. For models with too much initial sea ice, the bias-adjustment function ramps up from 


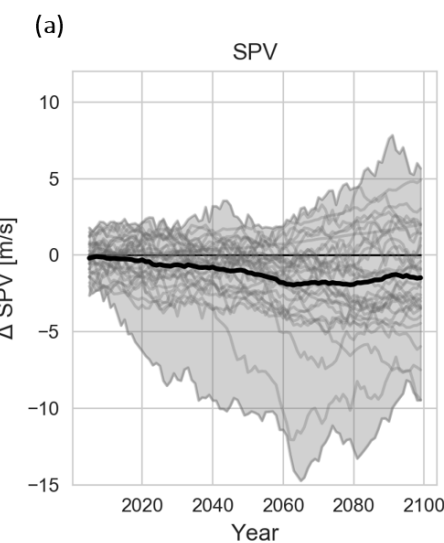

(d)

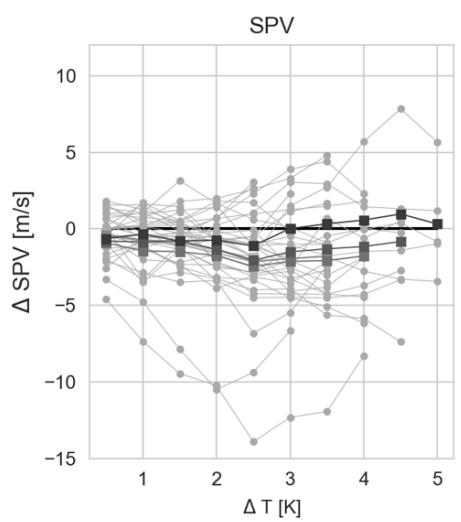

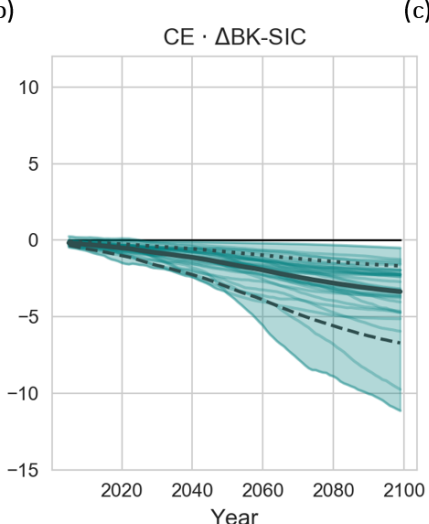

(e)

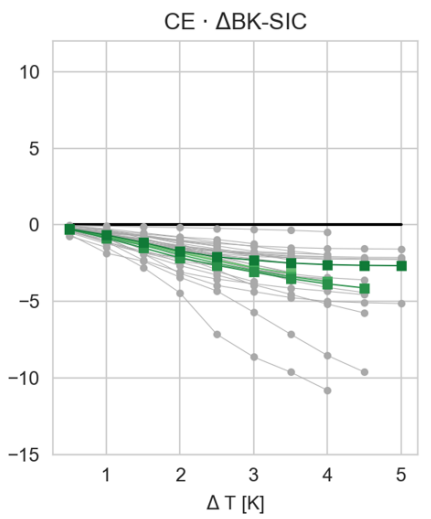

(c)

(f)
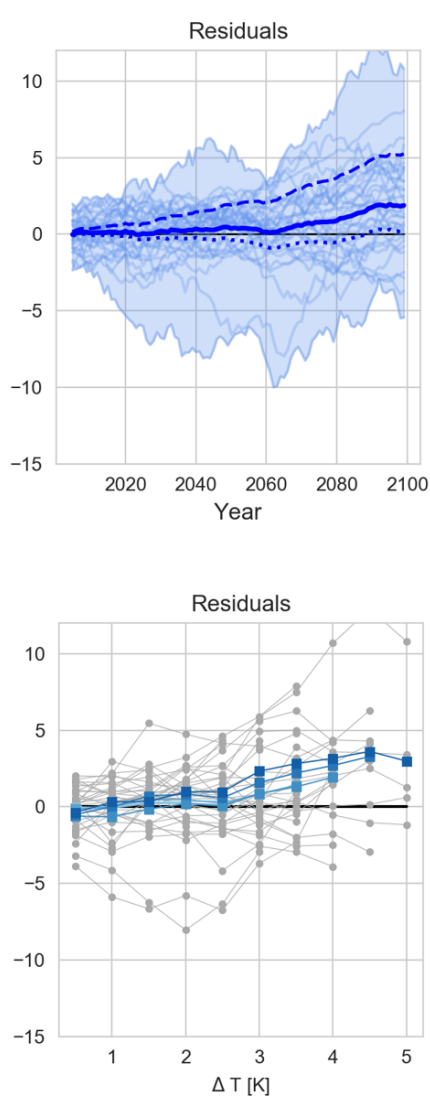

Figure 6. Decomposed projected polar vortex response. The 30-year running means of (a) projected SPV change, (b) BK-SIC-based predicted SPV change, and (c) the residuals of $(\mathbf{a}, \mathbf{b})$ as functions of time for a causal effect ce $=0.05$. The thick lines indicate the ensemble mean, and the dashed and dotted lines in (b) and (c) indicate the ensemble mean when using ce $=0.1$ and 0.025 , respectively. (d-f) Same as $(\mathbf{a}-\mathbf{c})$ but as a function of global mean temperature change and only for ce $=0.05$. The thick lines show the ensemble mean for different sub-sets of warming level reached at the end of the 21 st century with darker colours indicating higher warming levels.

zero when BK-SIC loss becomes unphysical, i.e. when it exceeds the observational BK-SIC, and stays constant as soon as the model's BK seas are ice-free. The constant is the erroneous amount of BK-SIC loss in that model multiplied by the specified causal effect $\mathrm{CE}_{\mathrm{m}}$ on SPV (see Fig. 5a). For models with too little initial sea ice, the bias-adjustment function ramps down from zero starting when the BK seas are ice-free and remains constant after when the model would have been ice-free had it had a realistic initial BK-SIC (see Methods).

Model 10 (CMCC-CESM), for example, exhibits large internal variability but shows a robust SPV weakening reaching almost $10 \mathrm{~m} \mathrm{~s}^{-1}$ at the end of the century (light blue line in Fig. 7c). However, this model has almost twice as much BK-SIC compared to observations, and thus the weakening response is likely overestimated. Bias adjusting this effect, starting when the BK-SIC loss exceeds the physically possible amount (see blue square in Fig. 7c), roughly halves the projected weakening at the end of the century (thick blue line Fig. 7c) when assuming a causal effect of 0.05 . An effect of 0.1 would imply almost no SPV change (dashed blue line in Fig. 7c), while that of 0.025 indicates a weakening of only around $7 \mathrm{~m} \mathrm{~s}^{-1}$ (dotted blue line in Fig. 7c).

In contrast, model 28 (MIROC-ESM) is the model with the most pronounced SPV strengthening of approximately $5 \mathrm{~m} \mathrm{~s}^{-1}$ at the end of the century (thin red line in Fig. 7c). Interestingly, the strengthening only starts after the BK seas have become ice-free (red square in Fig. 7c), which is consistent with the overall findings of this study. As the model has too little BK sea ice compared to observations, the projected pronounced strengthening is unrealistic. Adjusting for this bias leads to a strengthening of only about $2.5 \mathrm{~m} \mathrm{~s}^{-1}$ (thick red line in Fig. 7c) when assuming a causal effect of 0.05 and would even imply no change when assuming an effect of 0.1 (dashed red line in Fig. 7c).

Despite these large effects for individual models, the ensemble-mean-predicted SPV weakening from BK-SIC loss only marginally changes at the end of the century (see thick lines in Fig. 7d), while the spread is reduced by less 
(a)

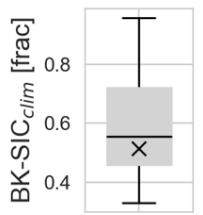

(b)

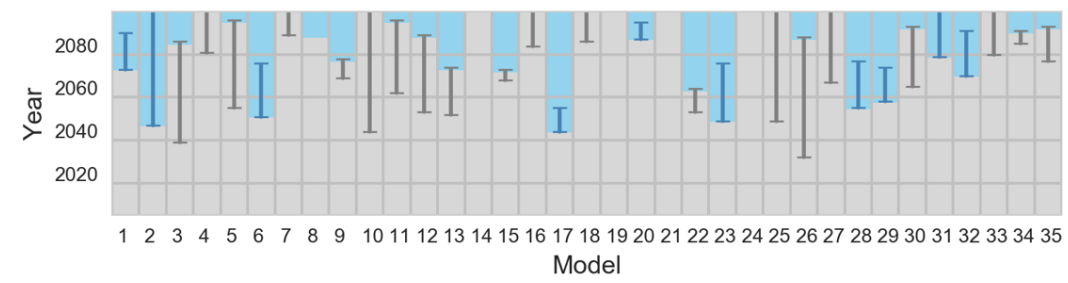

(c)

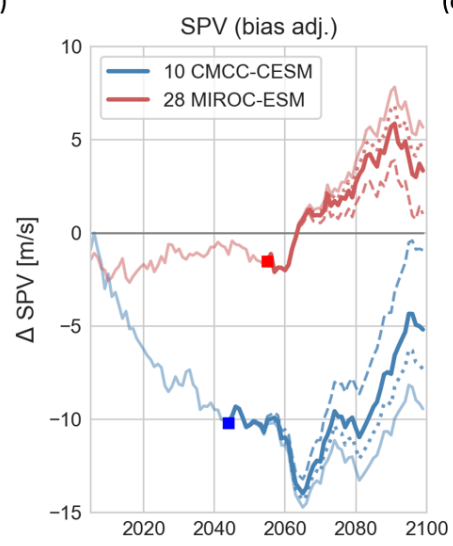

(d)

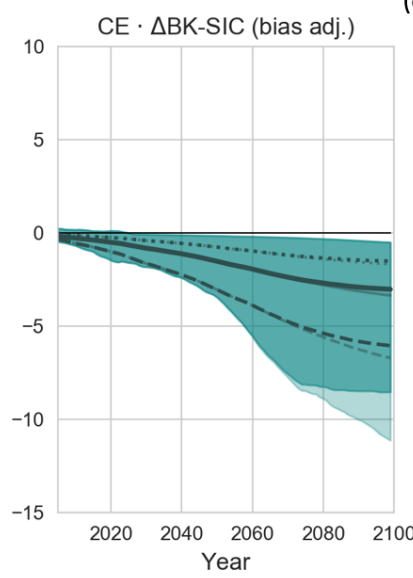

(e)

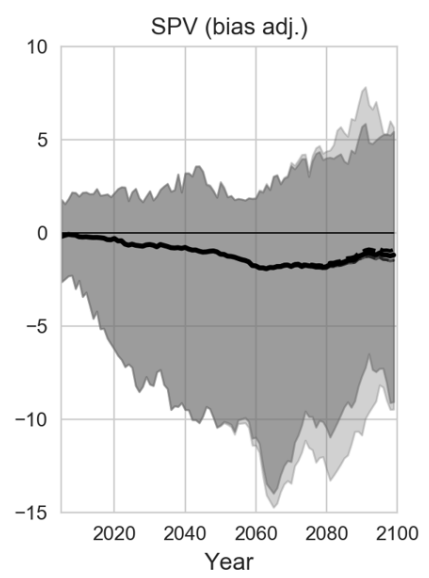

Figure 7. Bias-adjusted polar vortex change. (a) Spread across CMIP5 models of BK-SIC climatology (over 1960-1989 reference period). The black cross shows observational value, and the black line indicates the ensemble median. (b) Grey shading indicates years when BK seas are still ice covered, and blue shading indicates years after the BK seas have become ice-free (see methods). Vertical lines indicate the period over which the bias-adjustment function ramps either up or down, and grey indicates models with too much ice and blue models with too little ice. (c) Bias adjustment when assuming ce $=0.05$ (thick lines) for models 10 and 28. Light thin lines indicate the actual projected change, while dashed lines indicate bias adjustment when assuming a causal effect of ce $=0.1$ and dotted lines for ce $=0.025$. (d) Spread of predicted SPV from BK-SIC (light shading) and of bias-adjusted BK-SIC for ce $=0.05$ (dark shading). The dashed and dotted lines show the ensemble mean values for a causal effect of 0.1 and 0.025 , respectively. (e) Same as (d) but for projected SPV and for visualization reasons not showing the values for $c e=0.025$.

than one-third for a causal effect of 0.05 (Fig. 7d). Bias adjusting the initial sea ice conditions reduces the end-ofcentury ensemble mean (with model 5 and model 27 being excluded) from -1.4 to $-1.1 \mathrm{~m} \mathrm{~s}^{-1}$, lifts the lower bound from -9.5 to $-9 \mathrm{~m} \mathrm{~s}^{-1}$, and lowers the upper bound from 5.6 to $5.5 \mathrm{~m} \mathrm{~s}^{-1}$. Overall, the erroneous initial amount of BKSIC in the models thus does not substantially affect the projected SPV change (Fig. 7e).

\section{Discussion}

Our study adds to the large body of literature addressing the overarching question of whether the Arctic can, has, and will influence mid-latitude weather and climate (Barnes and Screen, 2015). Several previous studies have stressed the absence of a statistically significant signal to question claims concerning the influence of sea ice (Blackport and Screen, 2020; Seviour, 2017; Sun et al., 2016). However, absence of evidence is not evidence of absence (Shepherd, 2016). Moreover, if we reject the hypothesis of a causal influence, we are left with the puzzle of explaining the non-linearity seen in Figs. 1 and 2, whereas a weak causal influence is consistent with a lack of statistical significance. We have shown that despite the signal emerging from BK-SIC being likely small relative to internal variability and the proposed mediating pathway not being robustly identified in the CMIP5 ensemble, the impacts for the future SPV could nonetheless be pronounced due to the large loss of BK-SIC. In particular, assuming a weak influence of BK-SIC on SPV enables us to explain the disagreement on the sign of the SPV response to warming (Fig. 6) and a large part of the inter-model spread in the model projections (Fig. 5). Given that declining Arctic sea ice is certain in a warming climate, we argue for putting more focus on the avoidance of type-2 errors (Anderegg et al., 2014; Shepherd, 2019) to fully address the plausible range of regional climate impacts of Arctic sea ice loss (Sutton, 2019).

Quantifying the influence of BK-SIC on SPV is difficult. One potential reason is the documented non-stationarity of the Arctic-stratosphere pathway with several models exhibit- 
ing large decadal SPV variability (Kolstad and Screen, 2019; Siew et al., 2020). Another challenge we encountered is the choice of the relevant sea ice months, which seems to vary across models and might also change from year to year for a given model (Blackport and Screen, 2019; García-Serrano et al., 2017). For a fixed lag and month, only approximately half of the models show an expected negative link from BKSIC to Ural-SLP (Fig. 4b). On the other hand, using seasonal averages to estimate a net causal effect might have led to an underestimated causal effect in the models (Fig. 4a). An improved understanding of the timing of the BK-SIC to SPV pathway will be necessary to achieve progress.

The uncertainty regarding future SPV change contributes to uncertainty about future mid-latitude weather and climate (Simpson et al., 2018; Zappa and Shepherd, 2017). Our results of a weakened SPV in response to BK-SIC decrease are overall consistent with a reported equatorward jet shift and negative NAO-like response to sea ice loss across the CMIP5 models (Screen and Blackport, 2019; Screen et al., 2018; Zappa et al., 2018). In this context, it was further shown that models with sea ice loss have a weakened SPV in late winter, while those without sea ice loss have a strengthened SPV (see e.g. Fig. S5 of Zappa et al., 2018). Yet how much the stratospheric pathway discussed in this study contributes to this compared to other Arctic-related mechanisms not involving the stratosphere remains an open question (Kretschmer et al., 2016; Nakamura et al., 2016; Wu and Smith, 2016; Zhang et al., 2018b).

Identifying the processes causing a strengthening of the SPV is beyond the scope of the present study but is relevant to understand future polar vortex change. Changes in both vertical and horizontal wave activity propagation might play a role (Wu et al., 2019). For example, a deepened Aleutian Low, as favoured by decreasing sea ice in the Pacific sector, might contribute to such a strengthening (Hu et al., 2018; McKenna et al., 2017; Nishii et al., 2010) (as suggested here in Fig. 4f).

More generally, this study shows the benefits of a causal network approach to identify and quantify teleconnection signals in multi-model ensembles. Making the assumptions of the underlying causal model explicit transforms domain knowledge into mathematically testable objects and can guide the statistical analysis. Testing different hypotheses in this way is thus a logical next step to further constrain future SPV changes.

\section{Summary and conclusion}

We have provided evidence for the non-linear response of the SPV to global mean warming that is suggested to result from a weakening caused by sea ice loss in the BK seas and opposing effects (not specified in this study) which dominate the SPV response once the BK seas are ice-free. The timing of the latter varies substantially across models as a result of different sea ice climatologies and different warming rates.

A plausible quantification of this BK-SIC to SPV teleconnection in the historical simulations resulted in a standardized causal effect in the range of only about 0.05 , which helps explain why it is difficult to detect. Yet the implications of such a small causal effect for future SPV projections in the RCP8.5 scenario are notable due to the expected dramatic shrinking of Arctic sea ice; BK-SIC change can explain all of the projected ensemble-mean SPV weakening and up to almost half of the ensemble spread over the 21 st century.

We finally noted that most models include unrealistic sea ice conditions compared to observations, and thus also their SPV response to sea ice loss is unrealistic. Although adjusting for this bias only marginally reduces the ensemble mean and spread of the projected SPV changes, it has pronounced implications for particular models at both ends of the range of projected SPV changes.

Overall, our study gives another example of a tug of war between different effects of global warming on atmospheric circulation changes. Understanding and quantifying these opposing effects is crucial to reduce uncertainties about regional climate change scenarios.

Data availability. Data sets for this research are available in these in-text data citation references and the associated repositories. Observational sea ice data (Titchner and Rayner, 2014): https://www. metoffice.gov.uk/hadobs/hadisst2/data/download.html (last access: November 2020) (Met Office, 2020); ERA5 reanalysis data (Hersbach et al., 2020): https://doi.org/10.24381/cds.6860a573 (CDS, 2020); and CMIP5 data (Taylor et al., 2012): https://esgf-node.llnl. gov/search/cmip5/ (last access: November 2020) (WCRP, 2020).

Supplement. The supplement related to this article is available online at: https://doi.org/10.5194/wcd-1-715-2020-supplement.

Author contributions. MK analysed the data and led the writing. MK, GZ, and TGS designed the study and contributed to the writing.

Competing interests. The authors declare that they have no conflict of interest.

Acknowledgements. We thank ECMWF and the Hadley Centre for making the ERA5 and sea ice data publicly available. We further acknowledge the World Climate Research Programme's Working Group on Coupled Modelling, which is responsible for CMIP, and we thank the climate modelling groups listed in Fig. 4 for producing and making available their model outputs. Marlene Kretschmer has received funding from the European Union's Horizon 2020 research and innovation programme under the Marie SkłodowskaCurie grant agreement (no. 841902). Theodore G. Shepherd ac- 
knowledges support from ERC Advanced Grant 339390 (ACRCC). We thank the editor and the reviewers for their feedback which greatly helped to improve the paper.

Financial support. This research has been supported by the European Union's Horizon 2020 Marie Skłodowska-Curie (grant no. 841902) and the ERC Advanced Grant (grant no. 339390).

Review statement. This paper was edited by Tim Woollings and reviewed by two anonymous referees.

\section{References}

Anderegg, W. R. L., Callaway, E. S., Boykoff, M. T., Yohe, G., and Root, T. L.: Awareness of both type 1 and 2 errors in climate science and assessment, B. Am. Meteorol. Soc., 95, 1445-1451, https://doi.org/10.1175/BAMS-D-13-00115.1, 2014.

Ayarzaguiena, B., Charlton-Perez, A. J., Butler, A. H., Hitchcock, P., Simpson, I. R., Polvani, L. M., Butchart, N., Gerber, E. P., Gray, L., Hassler, B., Lin, P., Lott, F., Manzini, E., Mizuta, R., Orbe, C., Osprey, S., Saint-Martin, D., Sigmond, M., Taguchi, M., Volodin, E. M., and Watanabe, S.: Uncertainty in the Response of Sudden Stratospheric Warmings and StratosphereTroposphere Coupling to Quadrupled $\mathrm{CO}_{2}$ Concentrations in CMIP6 Models, J. Geophys. Res.-Atmos., 125, e2019JD032345, https://doi.org/10.1029/2019JD032345, 2020.

Baldwin, M. P. and Dunkerton, T. J.: Stratospheric harbingers of anomalous weather regimes, Science, 294, 581-584, https://doi.org/10.1126/science.1063315, 2001.

Barnes, E. A. and Screen, J. A.: The impact of Arctic warming on the midlatitude jet-stream: Can it? Has it? Will it?, Wiley Interdisciplin. Rev. Clim. Change, 6, 277-286, https://doi.org/10.1002/wcc.337, 2015.

Blackport, R. and Kushner, P. J.: Isolating the Atmospheric Circulation Response to Arctic Sea Ice Loss in the Coupled Climate System, J. Climate, 30, 2163-2185, https://doi.org/10.1175/JCLI-D16-0257.1, 2017.

Blackport, R. and Screen, J. A.: Influence of Arctic Sea Ice Loss in Autumn Compared to That in Winter on the Atmospheric Circulation, Geophys. Res. Lett., 46, 2213-2221, https://doi.org/10.1029/2018GL081469, 2019.

Blackport, R. and Screen, J. A.: Insignificant effect of Arctic amplification on the amplitude of midlatitude atmospheric waves, Sci. Adv., 6, eaay2880, https://doi.org/10.1126/sciadv.aay2880, 2020.

Blackport, R., Screen, J. A., van der Wiel, K., and Bintanja, R.: Minimal influence of reduced Arctic sea ice on coincident cold winters in mid-latitudes, Nat. Clim. Change, 9, 697-704, https://doi.org/10.1038/s41558-019-0551-4, 2019.

CDS: ERA5 monthly averaged data on pressure levels from 1979 to present, https://doi.org/10.24381/cds.6860a573, 2020.

Cohen, J., Zhang, X., Francis, J., Jung, T., Kwok, R., Overland, J., Ballinger, T. J., Bhatt, U. S., Chen, H. W., Coumou, D., Feldstein, S., Gu, H., Handorf, D., Henderson, G., Ionita, M., Kretschmer, M., Laliberte, F., Lee, S., Linderholm, H. W., Maslowski, W., Peings, Y., Pfeiffer, K., Rigor, I., Semmler, T., Stroeve, J., Taylor,
P. C., Vavrus, S., Vihma, T., Wang, S., Wendisch, M., Wu, Y., and Yoon, J.: Divergent consensuses on Arctic amplification influence on midlatitude severe winter weather, Nat. Clim. Change, 10, 20-29, https://doi.org/10.1038/s41558-019-0662-y, 2020.

$\mathrm{De}, \mathrm{B}$. and $\mathrm{Wu}, \mathrm{Y}$.: Robustness of the stratospheric pathway in linking the Barents-Kara Sea sea ice variability to the midlatitude circulation in CMIP5 models, Clim. Dynam., 53, 193207, https://doi.org/10.1007/s00382-018-4576-6, 2019.

de Vries, H., Woollings, T., Anstey, J., Haarsma, R. J., and Hazeleger, W.: Atmospheric blocking and its relation to jet changes in a future climate, Clim. Dynam., 41, 2643-2654, https://doi.org/10.1007/s00382-013-1699-7, 2013.

Domeisen, D. I. V., Garfinkel, C. I., and Butler, A. H.: The Teleconnection of El Niño Southern Oscillation to the Stratosphere, Rev. Geophys., 57, 5-47, https://doi.org/10.1029/2018RG000596, 2018.

García-Serrano, J., Frankignoul, C., King, M. P., Arribas, A., Gao, Y., Guemas, V., Matei, D., Msadek, R., Park, W., and SanchezGomez, E.: Multi-model assessment of linkages between eastern Arctic sea-ice variability and the Euro-Atlantic atmospheric circulation in current climate, Clim. Dynam., 49, 2407-2429, https://doi.org/10.1007/s00382-016-3454-3, 2017.

Garfinkel, C. I., Son, S.-W., Song, K., Aquila, V., and Oman, L. D.: Stratospheric variability contributed to and sustained the recent hiatus in Eurasian winter warming, Geophys. Res. Lett., 44, 374382, https://doi.org/10.1002/2016GL072035, 2017.

Hersbach, H., Bell, B., Berrisford, P., Hirahara, S., Horányi, A., Muñoz-Sabater, J., Nicolas, J., Peubey, C., Radu, R., Schepers, D., Simmons, A., Soci, C., Abdalla, S., Abellan, X., Balsamo, G., Bechtold, P., Biavati, G., Bidlot, J., Bonavita, M., Chiara, G., Dahlgren, P., Dee, D., Diamantakis, M., Dragani, R., Flemming, J., Forbes, R., Fuentes, M., Geer, A., Haimberger, L., Healy, S., Hogan, R. J., Hólm, E., Janisková, M., Keeley, S., Laloyaux, P., Lopez, P., Lupu, C., Radnoti, G., Rosnay, P., Rozum, I., Vamborg, F., Villaume, S., and Thépaut, J.: The ERA5 Global Reanalysis, Q. J. Roy. Meteorol. Soc., 146, 1999-2049, https://doi.org/10.1002/qj.3803, 2020.

Hoshi, K., Ukita, J., Honda, M., Iwamoto, K., Nakamura, T., Yamazaki, K., Dethloff, K., Jaiser, R., and Handorf, D.: Poleward eddy heat flux anomalies associated with recent Arctic sea ice loss, Geophys. Res. Lett., 44, 446-454, https://doi.org/10.1002/2016GL071893, 2017.

Hu, D., Guan, Z., Tian, W., and Ren, R.: Recent strengthening of the stratospheric Arctic vortex response to warming in the central North Pacific, Nat. Commun., 9, 1697, https://doi.org/10.1038/s41467-018-04138-3, 2018.

IPCC: IPCC Fifth Assessment Report, available at: https://www. ipcc.ch/report/ar5/ (last access: 6 October 2017), 2014.

Jiménez-Esteve, B. and Domeisen, D. I. V.: The Tropospheric Pathway of the ENSO-North Atlantic Teleconnection, J. Climate, 31, 4563-4584, https://doi.org/10.1175/JCLI-D-17-0716.1, 2018.

Karpechko, A. Y. and Manzini, E.: Arctic Stratosphere Dynamical Response to Global Warming, J. Climate, 30, 7071-7086, https://doi.org/10.1175/JCLI-D-16-0781.1, 2017.

Kidston, J., Scaife, A. A., Hardiman, S. C., Mitchell, D. M., Butchart, N., Baldwin, M. P., and Gray, L. J.: Stratospheric influence on tropospheric jet streams, storm tracks and surface weather, Nat. Geosci, 8, 433-440, https://doi.org/10.1038/ngeo2424, 2015. 
Kim, B.-M., Son, S.-W., Min, S.-K., Jeong, J.-H., Kim, S.-J., Zhang, X., Shim, T., and Yoon, J.-H.: Weakening of the stratospheric polar vortex by Arctic sea-ice loss, Nat. Commun., 5, 4646, https://doi.org/10.1038/ncomms5646, 2014.

Kolstad, E. W. and Screen, J. A.: Nonstationary Relationship Between Autumn Arctic Sea Ice and the Winter North Atlantic Oscillation, Geophys. Res. Lett., 46, 7583-7591, https://doi.org/10.1029/2019GL083059, 2019.

Kretschmer, M., Coumou, D., Donges, J. F., and Runge, J.: Using Causal Effect Networks to analyze different Arctic drivers of mid-latitude winter circulation, J. Climate, 29, 4069-4081, https://doi.org/10.1175/JCLI-D-15-0654.1, 2016.

Kretschmer, M., Coumou, D., Agel, L., Barlow, M., Tziperman, E., and Cohen, J.: More-Persistent Weak Stratospheric Polar Vortex States Linked to Cold Extremes, B. Am. Meteorol. Soc., 99, 4960, https://doi.org/10.1175/BAMS-D-16-0259.1, 2018.

Kug, J.-S., Jeong, J.-H., Jang, Y.-S., Kim, B.-M., Folland, C. K., Min, S.-K., and Son, S.-W.: Two distinct influences of Arctic warming on cold winters over North America and East Asia, Nat. Geosci., 8, 759-762, https://doi.org/10.1038/ngeo2517, 2015.

Manzini, E., Karpechko, A. Y., Anstey, J., Baldwin, M. P., Black, R. X., Cagnazzo, C., Calvo, N., Charlton-Perez, A., Christiansen, B., Davini, P., Gerber, E., Giorgetta, M., Gray, L., Hardiman, S. C., Lee, Y.-Y., Marsh, D. R., McDaniel, B. A., Purich, A., Scaife, A. A., Shindell, D., Son, S.-W., Watanabe, S., and Zappa, G.: Northern winter climate change: Assessment of uncertainty in CMIP5 projections related to stratospheretroposphere coupling, J. Geophys. Res.-Atmos., 119, 79797998, https://doi.org/10.1002/2013JD021403, 2014.

Manzini, E., Karpechko, A. Y., and Kornblueh, L.: Nonlinear Response of the Stratosphere and the North Atlantic-European Climate to Global Warming, Geophys. Res. Lett., 45, 4255-4263, https://doi.org/10.1029/2018GL077826, 2018.

Martius, O., Polvani, L. M., and Davies, H. C.: Blocking precursors to stratospheric sudden warming events, Geophys. Res. Lett., 36, L14806, https://doi.org/10.1029/2009GL038776, 2009.

McCusker, K. E., Fyfe, J. C., and Sigmond, M.: Twentyfive winters of unexpected Eurasian cooling unlikely due to Arctic sea-ice loss, Nat. Geosci., 9, 838-842, https://doi.org/10.1038/ngeo2820, 2016.

McGraw, M. C. and Barnes, E. A.: Memory Matters: A Case for Granger Causality in Climate Variability Studies, J. Climate, 31, 3289-3300, https://doi.org/10.1175/JCLI-D-17-0334.1, 2018.

McKenna, C. M., Bracegirdle, T. J., Shuckburgh, E. F., Haynes, P. H., and Joshi, M. M.: Arctic sea-ice loss in different regions leads to contrasting Northern Hemisphere impacts, Geophys. Res. Lett., 45, 945-954, https://doi.org/10.1002/2017GL076433, 2017

Met Office: HadISST.2.2.0.0 Data, available at: https://www. metoffice.gov.uk/hadobs/hadisst2/data/download.html, last access: November 2020.

Nakamura, T., Yamazaki, K., Iwamoto, K., Honda, M., Miyoshi, Y., Ogawa, Y., Tomikawa, Y., and Ukita, J.: The stratospheric pathway for Arctic impacts on midlatitude climate, Geophys. Res. Lett., 43, 3494-3501, https://doi.org/10.1002/2016GL068330, 2016.

Nishii, K., Nakamura, H., and Orsolini, Y. J.: Cooling of the wintertime Arctic stratosphere induced by the western $\mathrm{Pa}$ - cific teleconnection pattern, Geophys. Res. Lett., 37, L13805, https://doi.org/10.1029/2010GL043551, 2010.

Notz, D. and Stroeve, J.: The Trajectory Towards a Seasonally Ice-Free Arctic Ocean, Curr. Clim. Change Rep., 4, 407-416, https://doi.org/10.1007/s40641-018-0113-2, 2018.

Overland, J. E., Dethloff, K., Francis, J. A., Hall, R. J., Hanna, E., Kim, S.-J., Screen, J. A., Shepherd, T. G., and Vihma, T.: Nonlinear response of mid-latitude weather to the changing Arctic, Nat. Clim. Change, 6, 992-999, https://doi.org/10.1038/nclimate3121, 2016.

Pearl, J.: Linear Models: A Useful "Microscope" for Causal Analysis, J. Caus. Infer., 1, 155-170, https://doi.org/10.1515/jci-20130003, 2013.

Peings, Y.: Ural Blocking as a Driver of Early-Winter Stratospheric Warmings, Geophys. Res. Lett., 46, 5460-5468, https://doi.org/10.1029/2019GL082097, 2019.

Runge, J., Petoukhov, V., and Kurths, J.: Quantifying the strength and delay of climatic interactions: The ambiguities of cross correlation and a novel measure based on graphical models, J. Climate, 27, 720-739, 2014.

Screen, J. A.: Simulated Atmospheric Response to Regional and Pan-Arctic Sea Ice Loss, J. Climate, 30, 3945-3962, https://doi.org/10.1175/JCLI-D-16-0197.1, 2017a.

Screen, J. A.: The missing Northern European winter cooling response to Arctic sea ice loss, Nat. Commun., 8, 14603, https://doi.org/10.1038/ncomms14603, 2017b.

Screen, J. A. and Blackport, R.: How Robust is the Atmospheric Response to Projected Arctic Sea Ice Loss Across Climate Models?, Geophys. Res. Lett., 46, 11406-11415, https://doi.org/10.1029/2019GL084936, 2019.

Screen, J. A., Deser, C., Smith, D. M., Zhang, X., Blackport, R., Kushner, P. J., Oudar, T., McCusker, K. E., and Sun, L.: Consistency and discrepancy in the atmospheric response to Arctic sea-ice loss across climate models, Nat. Geosci., 11, 155-163, https://doi.org/10.1038/s41561-018-0059-y, 2018.

Seviour, W. J. M.: Weakening and shift of the Arctic stratospheric polar vortex: Internal variability or forced response?, Geophys. Res. Lett., 44, 3365-3373, https://doi.org/10.1002/2017GL073071, 2017.

Shepherd, T. G.: Atmospheric circulation as a source of uncertainty in climate change projections, Nat. Geosci., 7, 703-708, https://doi.org/10.1038/ngeo2253, 2014.

Shepherd, T. G.: Effects of a warming Arctic, Science, 353, 989990, https://doi.org/10.1126/science.aag2349, 2016.

Shepherd, T. G.: Storyline approach to the construction of regional climate change information, P. Roy. Soc. A, 475, 20190013, https://doi.org/10.1098/rspa.2019.0013, 2019.

Siew, P. Y. F., Li, C., Sobolowski, S. P., and King, M. P.: Intermittency of Arctic-mid-latitude teleconnections: stratospheric pathway between autumn sea ice and the winter North Atlantic Oscillation, Weather Clim. Dynam., 1, 261-275, https://doi.org/10.5194/wcd-1-261-2020, 2020.

Sigmond, M. and Scinocca, J. F.: The Influence of the Basic State on the Northern Hemisphere Circulation Response to Climate Change, J. Climate, 23, 1434-1446, https://doi.org/10.1175/2009JCLI3167.1, 2010.

Simpson, I. R., Hitchcock, P., Seager, R., Wu, Y., and Callaghan, P.: The Downward Influence of Uncertainty in the Northern Hemisphere Stratospheric Polar Vortex Response to Climate Change, 
J. Climate, 31, 6371-6391, https://doi.org/10.1175/JCLI-D-180041.1, 2018.

Smith, K. L., Polvani, L. M., and Tremblay, L. B.: The Impact of Stratospheric Circulation Extremes on Minimum Arctic Sea Ice Extent, J. Climate, 31, 7169-7183, https://doi.org/10.1175/JCLID-17-0495.1, 2018.

Sun, L., Deser, C., and Tomas, R. A.: Mechanisms of Stratospheric and Tropospheric Circulation Response to Projected Arctic Sea Ice Loss, J. Climate, 28, 7824-7845, https://doi.org/10.1175/JCLI-D-15-0169.1, 2015.

Sun, L., Perlwitz, J., and Hoerling, M.: What caused the recent "Warm Arctic, Cold Continents" trend pattern in winter temperatures?, Geophys. Res. Lett., 43, 5345-5352, https://doi.org/10.1002/2016GL069024, 2016.

Sutton, R. T.: Climate Science Needs to Take Risk Assessment Much More Seriously, B. Am. Meteorol. Soc., 100, 1637-1642, https://doi.org/10.1175/BAMS-D-18-0280.1, 2019.

Taylor, K. E., Stouffer, R. J., and Meehl, G. A.: An overview of CMIP5 and the experiment design, B. Am. Meteorol. Soc., 93, 485-498, https://doi.org/10.1175/BAMS-D-11-00094.1, 2012.

Titchner, H. A. and Rayner, N. A.: The Met Office Hadley Centre sea ice and sea surface temperature data set, version 2: 1 . Sea ice concentrations, J. Geophys. Res.-Atmos., 119, 2864-2889, https://doi.org/10.1002/2013JD020316, 2014.

Trenberth, K. E. and Hurrell, J. W.: Decadal atmosphereocean variations in the Pacific, Clim. Dynam., 9, 303-319, https://doi.org/10.1007/BF00204745, 1994.

Tyrlis, E., Manzini, E., Bader, J., Ukita, J., Nakamura, H., and Matei, D.: Ural Blocking Driving Extreme Arctic Sea Ice Loss, Cold Eurasia, and Stratospheric Vortex Weakening in Autumn and Early Winter 2016-2017, J. Geophys. Res.-Atmos., 124, 11313-11329, https://doi.org/10.1029/2019JD031085, 2019.

Warner, J. L., Screen, J. A., and Scaife, A. A.: Links Between Barents-Kara Sea Ice and the Extratropical Atmospheric Circulation Explained by Internal Variability and Tropical Forcing, Geophys. Res. Lett., 47, e2019GL085679, https://doi.org/10.1029/2019GL085679, 2020.
Waugh, D. W., Sobel, A. H., and Polvani, L. M.: What is the Polar Vortex, and how does it influence weather?, B. Am. Meteorol. Soc., 98, 37-44, https://doi.org/10.1175/BAMS-D-15-00212.1, 2016.

WCRP: Home page, available at: https://esgf-node.llnl.gov/search/ cmip5/, last access: November 2020.

Wu, Y. and Smith, K. L.: Response of Northern Hemisphere Midlatitude Circulation to Arctic Amplification in a Simple Atmospheric General Circulation Model, J. Climate, 29, 2041-2058, https://doi.org/10.1175/JCLI-D-15-0602.1, 2016.

Wu, Y., Simpson, I. R., and Seager, R.: Intermodel Spread in the Northern Hemisphere Stratospheric Polar Vortex Response to Climate Change in the CMIP5 Models, Geophys. Res. Lett., 46, 13290-13298, https://doi.org/10.1029/2019GL085545, 2019.

Zappa, G. and Shepherd, T. G.: Storylines of Atmospheric Circulation Change for European Regional Climate Impact Assessment, J. Climate, 30, 6561-6577, https://doi.org/10.1175/JCLI-D-160807.1, 2017.

Zappa, G., Pithan, F., and Shepherd, T. G.: Multimodel Evidence for an Atmospheric Circulation Response to Arctic Sea Ice Loss in the CMIP5 Future Projections, Geophys. Res. Lett., 45, 10111019, https://doi.org/10.1002/2017GL076096, 2018.

Zhang, P., Wu, Y., and Smith, K. L.: Prolonged effect of the stratospheric pathway in linking Barents-Kara Sea sea ice variability to the midlatitude circulation in a simplified model, Clim. Dynam., 50, 527-539, https://doi.org/10.1007/s00382-017-3624-y, 2018a.

Zhang, P., Wu, Y., Simpson, I. R., Smith, K. L., Zhang, X., De, B., and Callaghan, P.: A stratospheric pathway linking a colder Siberia to Barents-Kara Sea sea ice loss, Sci. Adv., 4, eaat6025, https://doi.org/10.1126/sciadv.aat6025, 2018b. 